\title{
miR-506 regulates breast cancer cell metastasis by targeting IQGAP1
}

\author{
GUANG SUN, YANXI LIU, KEREN WANG and ZHELI XU \\ Breast Surgical Department of China-Japan Union Hospital of Jilin University, Changchun, Jilin 130033, P.R. China
}

Received June 14, 2015; Accepted July 29, 2015

DOI: 10.3892/ijo.2015.3161

\begin{abstract}
MicroRNA (miRNA or miR)-506 is a novel miRNA related to the survival of breast cancer patients. However, the mechanism underlying miRNA-506 involvement in breast carcinogenesis remains unclear. In the present study, we found that miR-506 was downregulated in human breast malignant tissues and breast cancer cell lines by RT-qPCR analysis, and the expression level of miR-506 was decreased with the increasing of tumor stage. Subsequently, gain-of-function and loss-of-function experiments were performed in vitro, and the results from MTT assay, Transwell-Matrigel invasion assay and cell adhesion assay revealed that miR-506 suppresses cell proliferation, invasion and adhesion of breast cancer cells. Luciferase reporter assay revealed that IQ motif containing GTPase activating protein 1 (IQGAP1) is a direct target of miR-506. miR-506 represses the expression of IQGAP1 and its downstream extracellular signal regulated kinase (ERK) mitogen-activated protein kinase (MAPK) signaling pathways, as demonstrated by the RT-qPCR and western blot analysis. Furthermore, we found that IQGAP1 rescues the effect of miR-506 on cell proliferation, invasion, adhesion, and the activation of ERK MAPK signaling. In conclusion, the present study is the first to provide evidence that miR-506 acts as a tumor suppressor, at least partially, by directly downregulating IQGAP1 in breast cancer cells. The miR-506/IQGAP1/ERK pathway may be a novel therapeutic target in breast cancer.
\end{abstract}

\section{Introduction}

Breast cancer is the most commom malignancy in women worldwide (1-3). Similar to many other solid tumors, distant

Correspondence to: Professor Keren Wang or Professor Zheli Xu, Breast Surgical Department of China-Japan Union Hospital of Jilin University, 126 Xiantai Street, Changchun, Jilin 130033, P.R. China

E-mail: ke_ren_w@163.com

E-mail: xuzl2000@126.com

Key words: microRNA-506, breast cancer, metastasis, IQ motif containing GTPase activating protein 1 metastasis are responsible for $>90 \%$ of breast cancer-related mortality (4).

MicroRNAs (miRNAs or miRs) are a subclass of 19-25 nucleotides in length, non-coding RNAs that have received increasingly attention in recent years. miRNAs play important regulatory roles in a variety of biological processes, such as cellular proliferation, differentiation, apoptosis and motility (5). Growing evidence indicates that the alteration of miRNA expression in tumors is associated with tumor development and progression (6). miR-506 is a novel miRNA, and it has been demonstrated that the expression pattern of miR-506 is different in different types of malignant tumors, suggesting the role of miR-506 is complex in cancer progression (7-10). The meta-analysis revealed that miR-506 is related to the survival of breast cancer patients (11). However, the mechanism underlying miRNA-506 involvement in breast carcinogenesis remains unclear.

In the present study, we investigated the expression of miR-506 in different breast tissues and breast cancer cell lines. In addition, gain-of-function and loss-of-function experiments were performed in vitro to examine the role of miR-506 in breast cancer cell proliferation, invasion and adhesion. Furthermore, a novel target by which miR-506 exerts its effects on breast carcinogenesis was identified.

\section{Materials and methods}

Clinical breast tissues. The present study was approved by the Ethics Committee of the China-Japan Union Hospital of Jilin University. The surgical specimens, including 48 normal, 42 fibroadenoma and 48 malignant breast tissues were obtained from patients in the Breast Surgical Department of ChinaJapan Union Hospital of Jilin University. Among these 48 patients with malignant breast cancer, 8 patients had stage 0 , 13 patients had stage I, 10 patients had stage II, 9 patients had stage III and the other 8 patient had stage IV, at the time of the diagnosis. None of these patients had received chemotherapy or radiation therapy treatment before surgery. Written informed consent was obtained from each patient prior to enrollment in this study.

Cell culture and cell transfection. Human breast cancer cell lines (T47D, MDA-MB-231, MCF7, SK-BR-3 and HCC1937), normal human epithelial mammary cell line MCF-10A and HEK293 cells were obtained from the American Type 
Culture Collection (ATCC; Manassas, VA, USA) and grown in Dulbecco's modified Eagle's medium (DMEM; Invitrogen, Carlsbad, CA, USA) supplemented with $8 \%$ heat-inactivated fetal bovine serum (FBS; Invitrogen). The cells were maintained at $37^{\circ} \mathrm{C}$ in $5 \% \mathrm{CO}_{2}$ and were passaged every $2-3$ days. The miR-negative control (NC), miR-506 mimic, miR-506 inhibitor, IQGAP1-pcDNA3.1 and IQGAP1-shRNA were transfected into the cells using Lipofectamine 2000 (Invitrogen) according to the manufacturer's instructions.

Luciferase assay. Luciferase constructs were made by ligating fragments containing the wild-type (WT) and mutant-type (MUT) 3' untranslated region (UTR) of IQ motif containing GTPase activating protein 1 (IQGAP1) in pMIR-REPORT luciferase vector (Applied Biosystems, Foster City, CA, USA). The luciferase constructs were co-transfected with the miR-NC or the miR-506 mimic into the HEK293 cells. Firefly and Renilla luciferase activity were measured using the DualLuciferase reporter assay system (Promega, Madison, WI, USA).

MTT assay. Cells $\left(5 \times 10^{3}\right)$ were suspended and cultured in the 96-well plates overnight. Following transfection, $10 \mu 1 \mathrm{MTT}$ solution $(0.5 \mathrm{mg} / \mathrm{ml}$; Sigma, St. Louis, MO, USA) was added to each well, and the plates were incubated at $37^{\circ} \mathrm{C}$ for $4 \mathrm{~h}$. The formazan granules were dissolved using DMSO (Sigma), and then the absorbance rates were measured at $570 \mathrm{~nm}$ using a microplate reader (Infinite M200; Tecan Group Ltd., Männedorf, Switzerland).

Transwell-Matrigel invasion assay. The Transwell inserts (Corning Incorporated, Corning, NY, USA) precoated with Matrigel (BD Biosciences, Franklin Lakes, NJ, USA) was used to determine breast cancer cell invasion ability. Briefly, the cells were suspended in the FBS-free medium at the density of $5 \times 10^{4}$ cells $/ \mathrm{ml}$ and placed in the upper chambers. The lower chambers were filled with cell medium containing $8 \%$ FBS. Following incubation at $37^{\circ} \mathrm{C}$ for $24 \mathrm{~h}$, the cells on the upper surface of the membrane were removed by a cotton swab, and the cells on the lower surface of the insert were fixed in $95 \%$ ethanol and stained with hematoxylin. The invaded cells were counted under a light microscope (Nikon, Tokyo, Japan).

Cell adhesion assay. For adhesion assay, the 96-well plates were precoated with fibronectin (Sigma), and then blocked with $1 \%$ bovine serum albumin (BSA; Sigma) for $2 \mathrm{~h}$. The cells were suspended in the FBS-free medium at the density of $3 \times 10^{5}$ cells/ml and seeded in the 96-well plates. Following incubation at $37^{\circ} \mathrm{C}$ for $2 \mathrm{~h}$, the adhesive cells were fixed in $4 \%$ paraformaldehyde and stained with $0.5 \%$ crystal violet. The crystals were dissolved using SDS (Amresco LLC, Solon, OH, USA) and then the absorbance rates were measured at $570 \mathrm{~nm}$ using a microplate reader (Infinite M200; Tecan Group Ltd.).

Reverse transcription-quantitative polymerase chain reaction (RT-qPCR). Total RNA from the breast tissues and breast cancer cell lines was extracted using the TRIzol reagent (Invitrogen). For miR-506 amplification, mirVana RNA isolation kit (Ambion, Austin, TX, USA) was used for RNA isolation following the manufacturer's instructions. RNA samples were reverse transcribed to complementary DNAs using the RevertAid First Strand cDNA Synthesis kit (Fermentas, Vilnius, Lithuania). Subsequently, the cDNA was amplified by real-time PCR on an ABI Prism 7500 Fast Real-Time PCR system (Applied Biosystems) using the SYBR-Green PCR kit (Applied Biosystems). The conditions for PCR amplification were as follows: an initial $95^{\circ} \mathrm{C}$ for $5 \mathrm{~min}$, followed by 40 cycles of $95^{\circ} \mathrm{C}$ for $15 \mathrm{sec}, 58^{\circ} \mathrm{C}$ for $30 \mathrm{sec}$, and $72^{\circ} \mathrm{C}$ for $30 \mathrm{sec}$. The $\mathrm{Ct}$ value was calculated using the $\Delta \Delta \mathrm{Ct}$ method.

Western blot analysis. The total protein was extracted using the Total Protein Extraction kit (BioChain Institute Inc., Hayward, CA, USA). Protein $(30 \mu \mathrm{g})$ was resolved on $10 \%$ sodium dodecyl sulphate-polyacrylamide gel electrophoresis (SDS-PAGE) and transferred onto a nitrocellulose membrane (Millipore, Billerica, MA, USA) by electroblotting. The membranes were then incubated with 5\% BSA (Sigma) at $4^{\circ} \mathrm{C}$ overnight. After washing in Tris-buffered saline with $0.1 \%$ Tween-20, the membranes were incubated with the primary antibodies, including rabbit polyclonal to IQGAP1 (1:800; cat. sc-10792; Santa Cruz Biotechnology, Santa Cruz, CA, USA), rabbit polyclonal to B-Raf (1:400; cat. sc-9002; Santa Cruz Biotechnology), rabbit monoclonal to Erk1/2 (Thr202/Tyr204) (1:800; cat. 4695; Cell Signaling Technology, Beverly, MA, USA), rabbit monoclonal to phospho-Erk1/2 (Thr202/Tyr204) (1:500; cat. 4376; Cell Signaling Technology), and rabbit polyclonal to GAPDH (1:1,000; cat. sc-25778; Santa Cruz Biotechnology) at $37^{\circ} \mathrm{C}$ for $2 \mathrm{~h}$, followed by the incubation of horseradish peroxidase-conjugated goat anti-rabbit $\operatorname{IgG}$ (1:2,000; cat. sc-2004; Santa Cruz Biotechnology) at $37^{\circ} \mathrm{C}$ for $1 \mathrm{~h}$. Chemiluminescence detection was carried out by using ECL Plus $^{\mathrm{TM}}$ (GE Healthcare, Piscataway, NJ, USA).

Statistical analysis. All data were expressed as the mean \pm SD. Differences between 2 groups were assessed by the Student's t-test. Analysis was performed using SPSS 19.0 statistical software (SPSS, Inc., Chicago, IL, USA). A P-value $<0.05$ was considered significant.

\section{Results}

Expression of miR-506 in human breast tissues and breast cancer cell lines. Expression of miR-506 in the normal, fibroadenoma and malignant breast tissues was analyzed using RT-qPCR. The results showed that miR-506 expression was not significantly different between the normal and fibroadenoma breast tissues. However, miR-506 expression was significantly reduced in the malignant breast tissues compared with the normal and fibroadenoma breast tissues (Fig. 1A). Furthermore, we found that miR-506 expression was decreased with the increasing of tumor stage (Fig. 1B).

We detected the expression of miR-506 in five breast cancer cell lines (T47D, MDA-MB-231, MCF7, SK-BR-3 and HCC1937). A normal human epithelial mammary cell line MCF-10A was used as control. The results obtained from RT-qPCR analysis revealed that compared with the control, miR-506 expression was significantly decreased in the breast cancer cell lines. Among these breast cancer cell lines, miR-506 had the lowest expression in MDA-MB-231 cells, and highest expression in HCC1937 cells (Fig. 1C). 

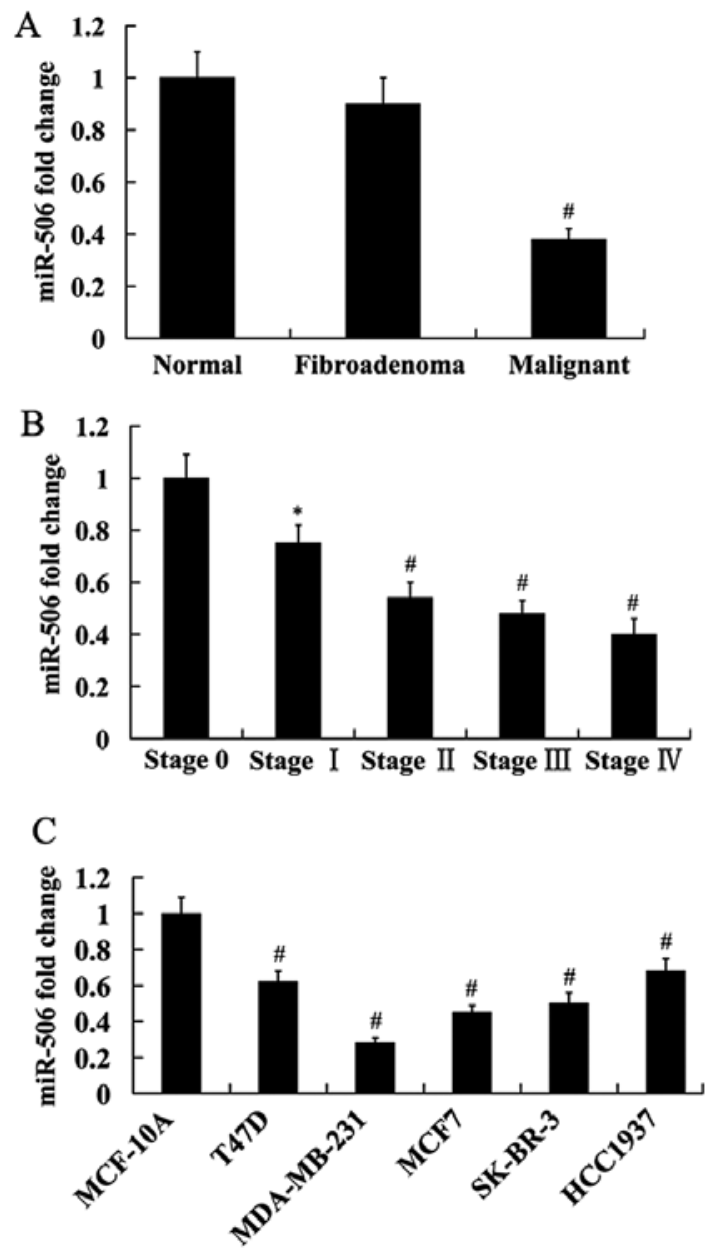

Figure 1. Expression of miR-506 in human breast tissues and breast cancer cell lines. (A) Fold changes of miR-506 in human breast tissues. ${ }^{~} \mathrm{P}<0.01$ compared with the normal. (B) Fold changes of miR-506 in malignant breast cancer patients with different tumor stage. ${ }^{*} \mathrm{P}<0.05$ and ${ }^{*} \mathrm{P}<0.01$ compared with stage 0. (C) Fold changes of miR-506 in human breast cancer cell lines and the normal human epithelial mammary cell line MCF-10A. ${ }^{\#} \mathrm{P}<0.01$ compared with MCF-10A.

Expression of IQGAPI in human breast tissues and breast cancer cell lines. IQGAP1 expression was also examined in human breast tissues and breast cancer cell lines. In contrast to miR-506 expression, IQGAP1 protein was significantly upregulated in the malignant breast tissues compared with the normal and fibroadenoma breast tissues (Fig. 2A). In addition, IQGAP1 protein was significantly increased in the breast cancer cell lines compared with the normal control MCF-10A cells (Fig. 2B).

Effect of miR-506 on cell proliferation, invasion and adhesion of breast cancer cells. To reveal the effect of miR-506 on breast cancer cell proliferation, invasion and adhesion, the miR-506 mimic was transfected into the MDA-MB-231 cells, which have low endogenous miR-506 expression, to overexpress miR-506. In addition, we knocked down the expression of miR-506 by transfection of the miR-506 inhibitor into the HCC1937 cells, which show high miR-506 expression. As demonstrated in Fig. 3A, the expression level of miR-506 was increased $~ 4.6$-fold in MDA-MB-231 cells following transfection with the miR-506 mimic; however, miR-506 expression
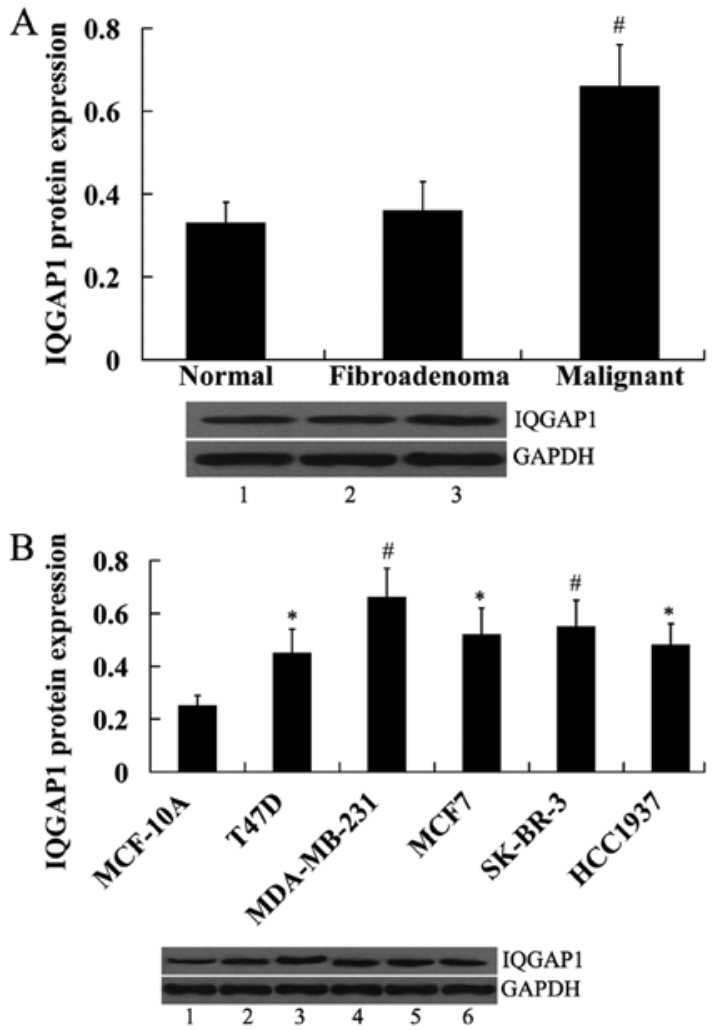

Figure 2. Expression of IQGAP1 in human breast tissues and breast cancer cell lines. (A) Expression of IQGAP1 protein in human breast tissues. ${ }^{\#} \mathrm{P}<0.01$ compared with the normal. Lane 1, normal; lane 2, fibroadenoma; lane 3, malignant. (B) Expression of IQGAP1 protein in human breast cancer cell lines and the normal human epithelial mammary cell line MCF-10A. The expression of IQGAP1 was normalized to GAPDH. Lanes 1-6 represent MCF-10A, T47D, MDA-MB-231, MCF7, SK-BR-3 and HCC1937, respectively. ${ }^{*} \mathrm{P}<0.05$ and ${ }^{\#} \mathrm{P}<0.01$ compared with MCF-10A. IQGAP1, IQ motif containing GTPase activating protein 1.
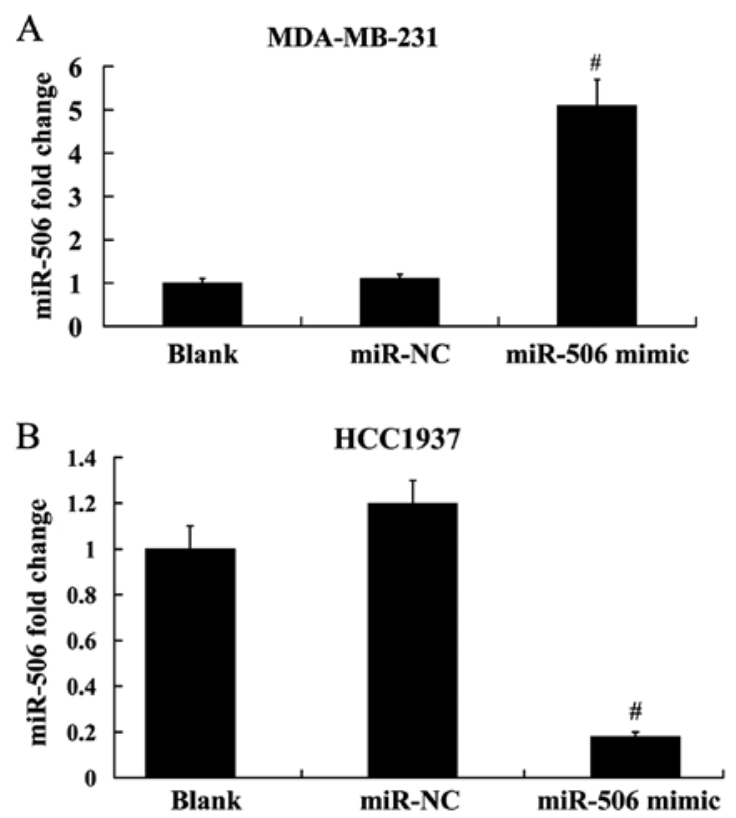

Figure 3. Expression of miR-506 in human breast cancer cells following transfection with the miR-506 mimic or the miR-506 inhibitor. (A) Fold changes of miR-506 in MDA-MB-231 cells following transfection with the miR-506 mimic. (B) Fold changes of miR-506 in HCC1937 cells following transfection with the miR-506 inhibitor. ${ }^{\#} \mathrm{P}<0.01$ compared with the miR-NC. NC, negative control. 

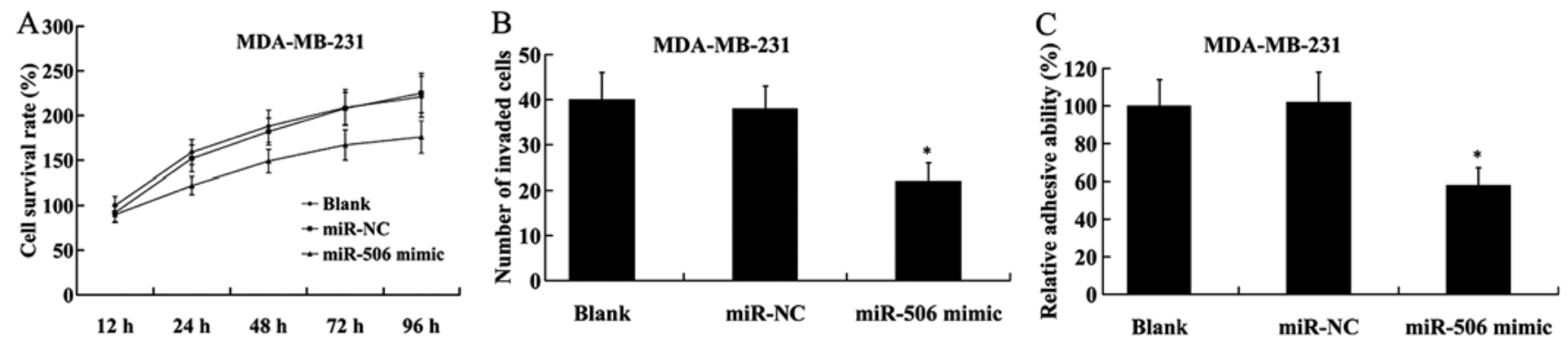

Figure 4. Effect of miR-506 overexpression on MDA-MB-231 cell proliferation, invasion and adhesion. (A) Effect of miR-506 overexpression on cell proliferation. (B) Effect of miR-506 overexpression on cell invasion. (C) Effect of miR-506 overexpression on cell adhesion. "P<0.05 compared with the miR-NC. NC, negative control.
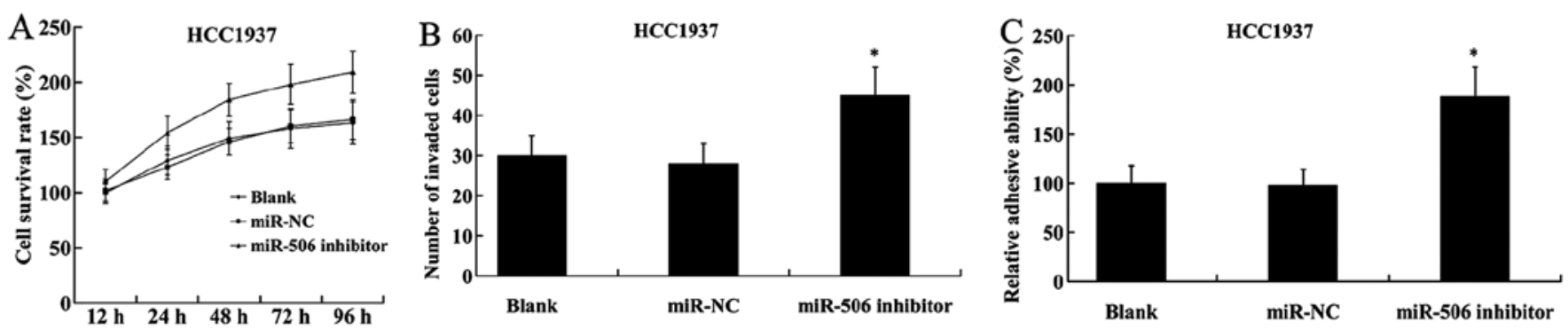

Figure 5. Effect of miR-506 suppression on HCC1937 cell proliferation, invasion and adhesion. (A) Effect of miR-506 suppresion on cell proliferation. (B) Effect of miR-506 suppression on cell invasion. (C) Effect of miR-506 suppression on cell adhesion. " $\mathrm{P}<0.05$ compared with the miR-NC. NC, negative control.
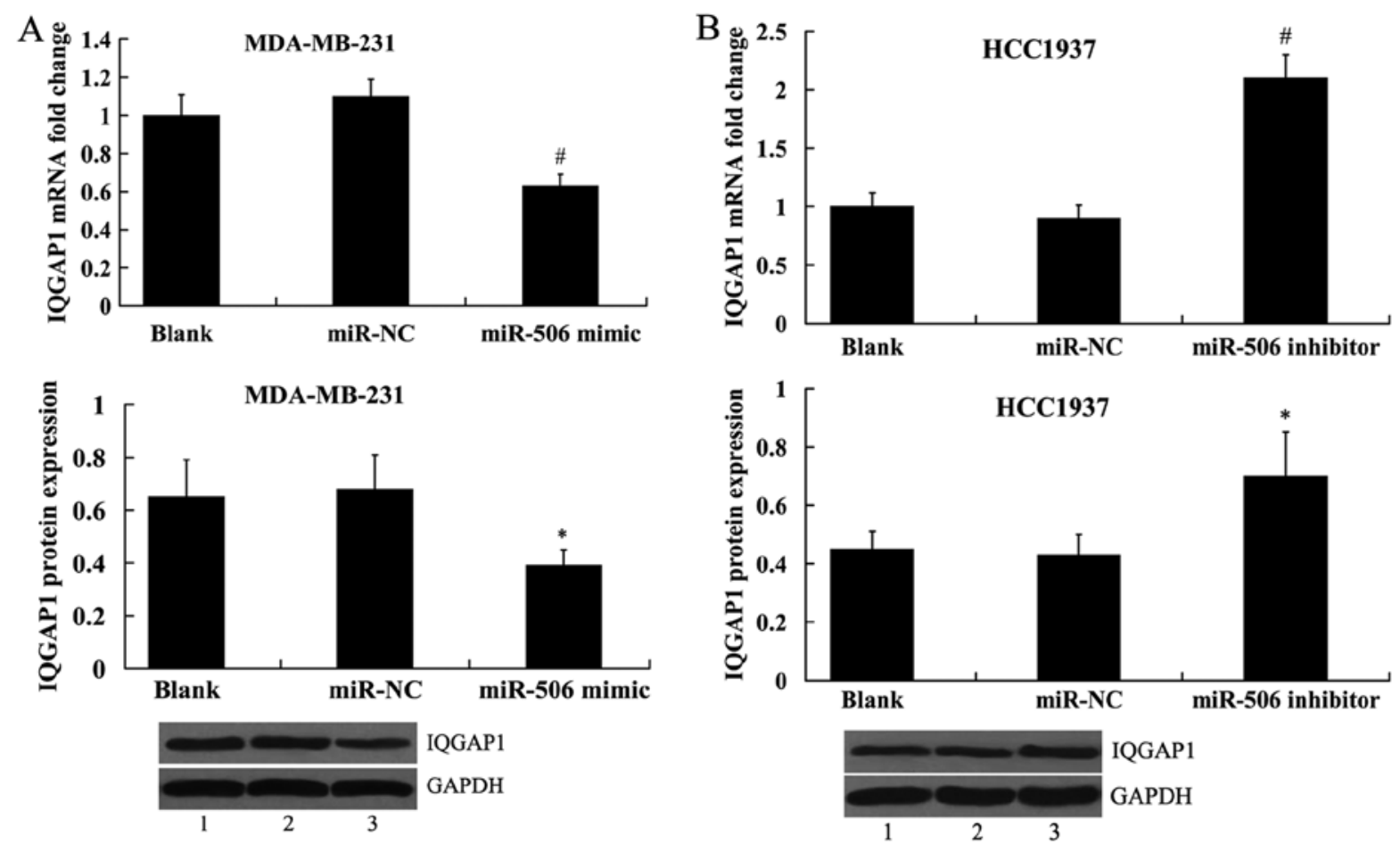

Figure 6. Effect of miR-506 on IQGAP1 mRNA and protein expression in breast cancer cells. (A) Effect of miR-506 overexpression on IQGAP1 mRNA and protein expression in MDA-MB-231 cells. Lane 1, blank; lane 2, miR-NC; lane 3, miR-506 mimic. (B) Effect of miR-506 suppression on IQGAP1 mRNA and protein expression in HCC1937 cells. The expression of IQGAP1 was normalized to GAPDH. Lane 1, blank; lane 2, miR-NC; lane 3, miR-506 inhibitor. ${ }^{*} \mathrm{P}<0.05$ and ${ }^{\text { }} \mathrm{P}<0.01$ compared with the miR-NC. NC, negative control; IQGAP1, IQ motif containing GTPase activating protein 1.

was decreased $\sim 85 \%$ in HCC1937 cells following transfection with the miR-506 inhibitor (Fig. 3B).
We found that compared with the control, MDA-MB-231 cells with ectopic expression of miR-506 showed a significant 

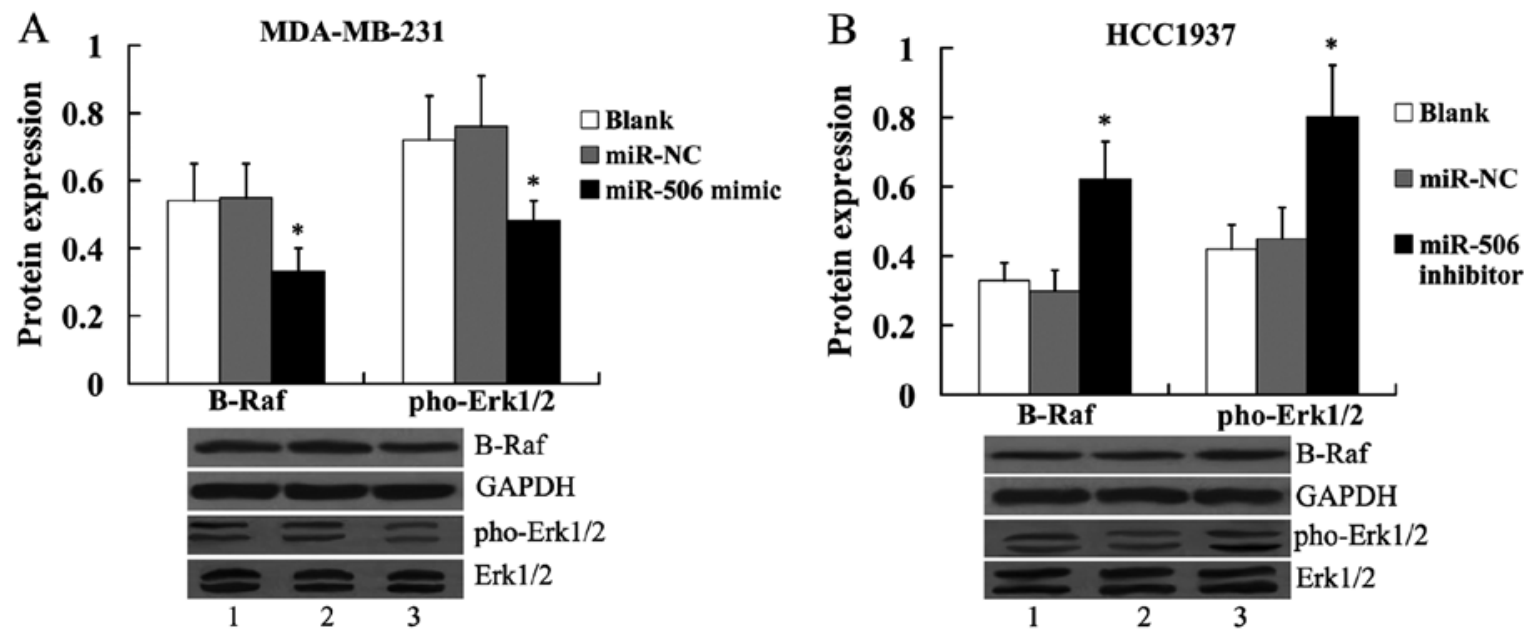

Figure 7. Effect of miR-506 on B-Raf and pho-Erk1/2 protein expression in breast cancer cells. (A) Effect of miR-506 overexpression on B-Raf and pho-Erk1/2 protein expression in MDA-MB-231 cells. Lane 1, blank; lane 2, miR-NC; lane 3, miR-506 mimic. (B) Effect of miR-506 suppression on B-Raf and pho-Erk1/2 protein expression in HCC1937 cells. The expression of B-Raf was normalized to GAPDH, while the expression of pho-Erk1/2 was normalized to total Erk1/2. Lane 1, blank; lane 2, miR-NC; lane 3, miR-506 inhibitor. * $\mathrm{P}<0.05$ compared with the miR-NC. NC, negative control; IQGAP1, IQ motif containing GTPase activating protein 1; Erk, extracellular signal regulated kinase; pho, phosphorylated.

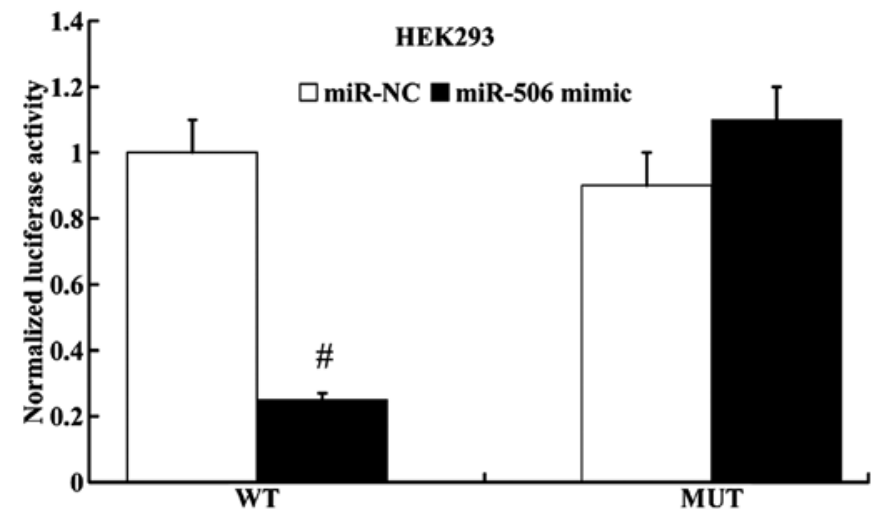

Figure 8. The relative luciferase activity of IQGAP1 wt or mut 3'UTR in HEK293 cells following transfection with the miR-506 mimic. ${ }^{*} \mathrm{P}<0.01 \mathrm{com}-$ pared with the miR-NC. NC, negative control; IQGAP1, IQ motif containing GTPase activating protein 1 .

decrease in cell proliferation by MTT assay (Fig. 4A). The number of invaded cells was also decreased in MDA-MB-231 cells transfected with the miR-506 mimic in comparison to the cells transfected with the miR-NC (Fig. 4B). Furthermore, cell adhesion assay revealed that following transfection with the miR-506 mimic, the adhesive ability of the MDA-MB-231 cells was significantly reduced compared with that of the miR-NC (Fig. 4C).

On the contrary, inhibition of miR-506 in HCC1937 cells led to the induction of cell proliferation, increased number of invaded cells and adhesive ability (Fig. 5).

Effect of miR-506 on IQGAP1, B-Raf and phosphorylated (pho)-extracellular signal regulated kinase (Erk) 1/2 expression. To investigate whether miR-506 regulates IQGAP1 expression, the miR-506 mimic and the miR-506 inhibitor was transfected into the MDA-MB-231 and HCC1937 cells, respectively, and IQGAP1 expression was analyzed both at the mRNA level and protein level. It was shown that IQGAP1 expression was significantly decreased in the MDA-MB-231 cells transfected with the miR-506 mimic (Fig. 6A). Transfection of miR-506 inhibitor into the HCC1937 cells led to increased expression of IQGAP1 (Fig. 6B).

In addition, we found that the ERK MAPK pathway was suppressed in MDA-MB-231 cells by transfection of the miR-506 mimic, as evidenced by the decreased expression of B-Raf and pho-Erk1/2 (Fig. 7A). In HCC1937 cells, the expression of B-Raf and pho-Erk1/2 was significantly increased by the miR-506 inhibitor (Fig. 7B).

miR-506 regulates IQGAPI expression by directly targeting its 3'UTR. miRanda algorithms were employed to search for putative gene targets of miR-506, and IQGAP1 was identified as a potential target. Subsequently, luciferase reporter assay was performed to determine whether IQGAP1 was a direct target of miR-506. IQGAP1 3'UTR (wt 3'UTR or mut 3'UTR) luciferase reporter vector was co-transfected with the miR-506 mimic or miR-NC into the HEK293 cells. As shown in Fig. 8, the luciferase activity of IQGAP1 wt 3'UTR was notably decreased in the miR-506 mimic group compared with the miR-NC group. However, the luciferase activity of IQGAP1 mut 3'UTR had no significant difference between the miR-506 mimic group and the miR-NC group.

IQGAPI attenuates the effect of miR-506 on cell proliferation, invasion and adhesion of breast cancer cells. Since IQGAP1 was shown to be a direct target of miR-506, its principle role was further investigated by its overexpression or suppression in MDA-MB-231 and HCC1937 cells in the presence of the miR-506 mimic or the miR-506 inhibitor. As shown in Fig. 9A, the expression of IQGAP1 was significantly increased in MDA-MB-231 cells following transfection with the miR-506 mimic and IQGAP1-pcDNA3.1 compared with the cells only transfected with the miR-506 mimic. In addition, transfection of the IQGAP1 shRNA in HCC1937 cells led to significantly decreased expression of IQGAP1 (Fig. 9B). 

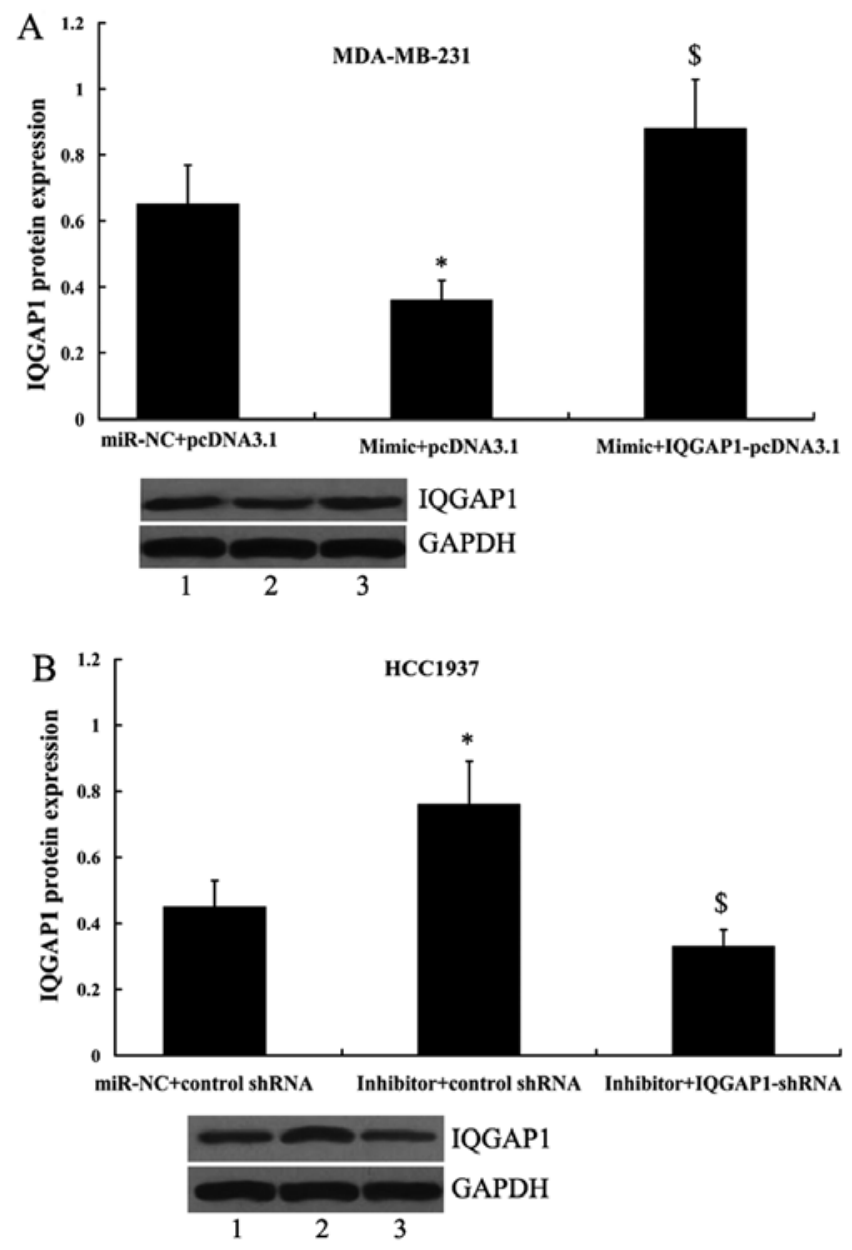

Figure 9. Expression of IQGAP1 protein in breast cancer cells following transfection with the miR-506 mimic/inhibitor and IQGAP1 pcDNA3.1/ shRNA. (A) Expression of IQGAP1 protein in MDA-MB-231 cells following transfection with the miR-506 mimic and IQGAP1-pcDNA3.1. Lane 1, miR-NC+pcDNA3.1; lane 2, mimic+ pcDNA3.1; lane 3, mimic+IQGAP1pcDNA3.1. ${ }^{*} \mathrm{P}<0.05$ compared with the miR-NC+pcDNA3.1; ${ }^{\mathrm{S}} \mathrm{P}<0.01$ compared with the mimic+pcDNA3.1. (B) Expression of IQGAP1 protein in HCC1937 cells following transfection with the miR-506 inhibitor and IQGAP1 shRNA. Lane 1, miR-NC+pcDNA3.1; lane 2, inhibitor+control shRNA; lane 3, inhibitor+IQGAP1-shRNA. "P<0.05 compared with the miR-NC+control shRNA; ${ }^{\$} \mathrm{P}<0.01$ compared with the inhibitor+control shRNA. The expression of IQGAP1 was normalized to GAPDH. NC, negative control; IQGAP1, IQ motif containing GTPase activating protein 1.

We found that the inhibition of cell proliferation, invasion and adhesion as a consequence of miR-506 mimic transfection in MDA-MB-231 cells was attenuated by the overexpression of IQGAP1 (Fig. 10).

As expected, IQGAP1 suppression also reversed the effect of miR-506 inhibitor on cell proliferation, invasion and adhesion in HCC1937 cells (Fig. 11).

IQGAP1 attenuates the effect of miR-506 on B-Raf and pho-Erk1/2 expression. In addition, we found that the inhibitory effect of miR-506 mimic on the expression of B-Raf and pho-Erk1/2 in MDA-MB-231 cells could be reversed in the presence of IQGAP1 overexpression (Fig. 12A). However, the increased expression of B-Raf and pho-Erk1/2 by the transfection of miR-506 inhibitor was abolished by IQGAP1-shRNA (Fig. 12B).
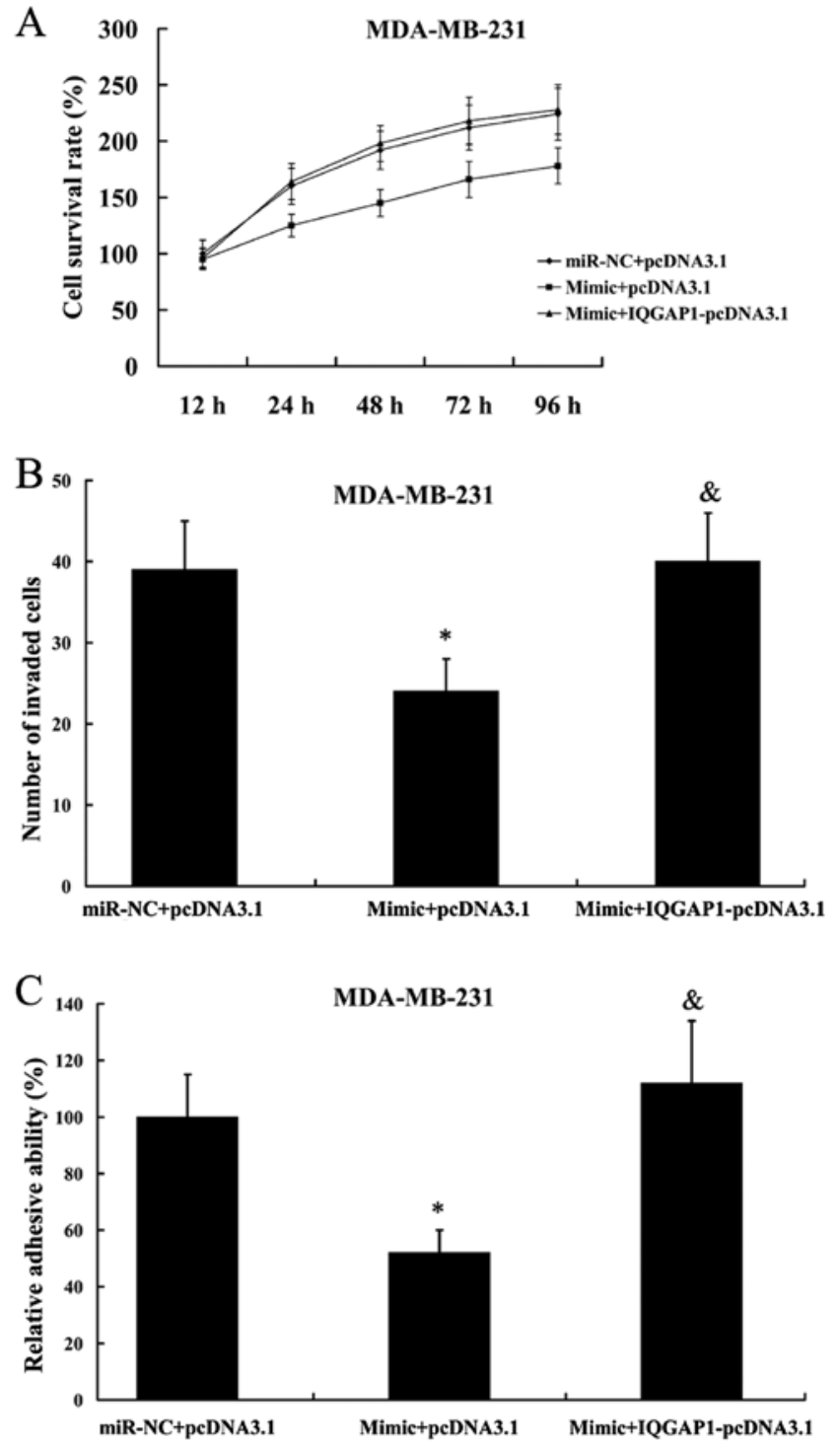

Figure 10. IQGAP1 overexpression attenuated the effect of miR-506 on MDA-MB-231 cell proliferation, invasion and adhesion. (A) IQGAP1 overexpression attenuated the effect of miR-506 on cell proliferation. (B) IQGAP1 overexpression attenuated the effect of miR-506 on cell invasion. (C) IQGAP1 overexpression attenuated the effect of miR-506 on cell adhesion. ${ }^{*} \mathrm{P}<0.05$ compared with miR-NC+pcDNA3.1; ${ }^{\text {\& }} \mathrm{P}<0.05$ compared with mimic+pcDNA3.1. NC, negative control; IQGAP1, IQ motif containing GTPase activating protein 1 .

\section{Discussion}

In the present study, we demonstrated downregulation of miR-506 in both the breast malignant tissues and human breast cancer cell lines. In addition, expression level of miR-506 was decreased with the increasing of tumor stage. These results indicate that miR-506 is clearly involved in the development of human breast cancer. Subsequently, in vitro experiments were performed to investigate the role of miR-506 on breast cancer cell proliferation, invasion and adhesion.

miR-506 was cloned relatively recently, and it acts as a tumor suppressive miRNA in various cancers and malignantly transformed cells $(7-9,12,13)$. Downregulation of miR-506 has been identified in various tumors, and overexpression 
A

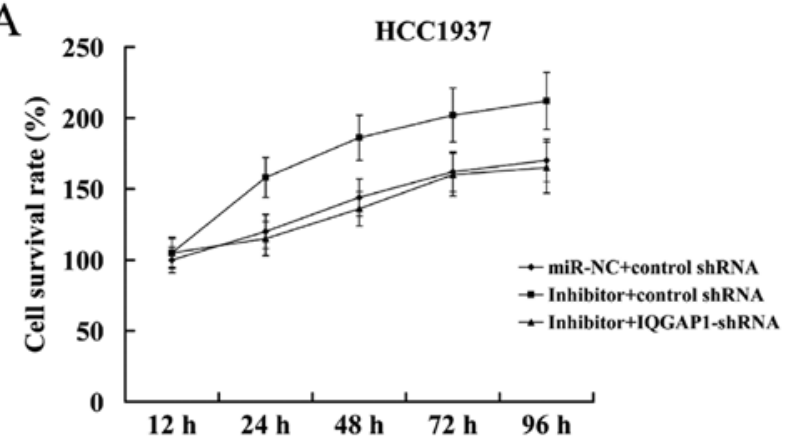

B

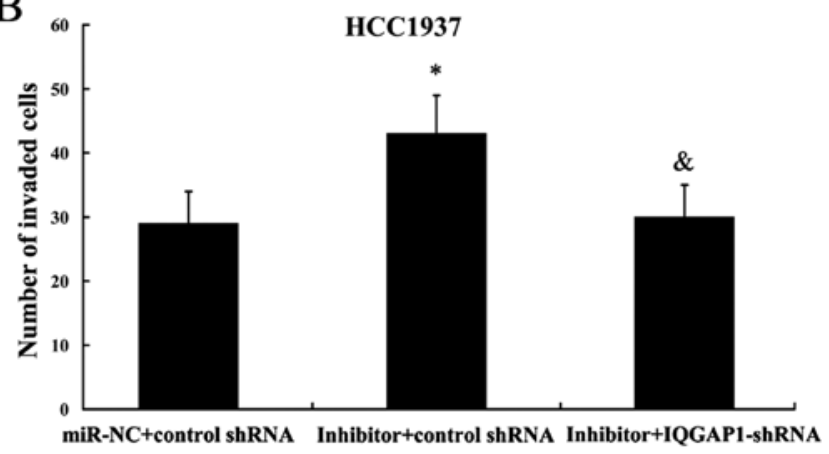

$\mathrm{C}$

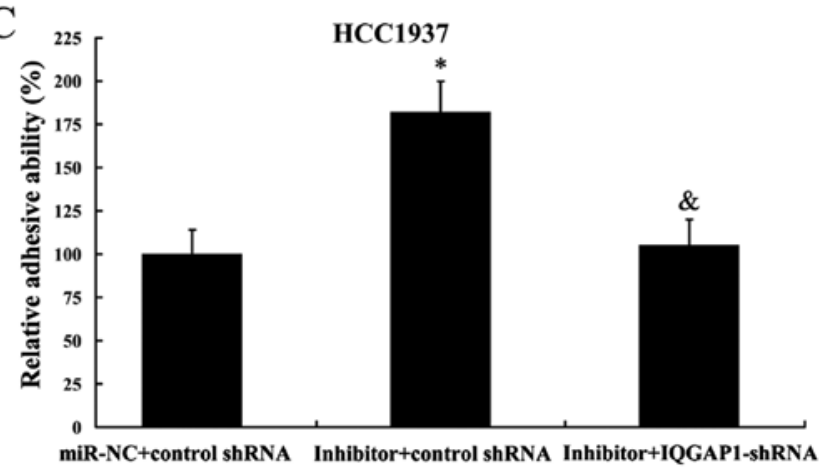

Figure 11. IQGAP1 suppression attenuates the effect of miR-506 on HCC1937 cell proliferation, invasion and adhesion. (A) IQGAP1 suppression attenuated the effect of miR-506 on cell proliferation. (B) IQGAP1 suppression attenuated the effect of miR-506 on cell invasion. (C) IQGAP1 suppression attenuated the effect of miR-506 on cell adhesion. ${ }^{*} \mathrm{P}<0.05$ compared with miR-NC+control shRNA; ${ }^{\&} \mathrm{P}<0.05$ compared with inhibitor+control shRNA. NC, negative control; IQGAP1, IQ motif containing GTPase activating protein 1 .

of miR-506 shows inhibitory effect on the development and progression of hepatocellular carcinoma, cervical cancer and ovarian cancer $(7,9,13)$. However, Streicher et al (10) reported that miR-506 was upregulated and acts as an oncogene in melanomas. Taken together, these findings suggested that the function of miR-506 appears to be cell type-specific. In the present study, we clearly demonstrated the anti-oncogenic role of miR-506 in breast cancer cells. Both gain-of-function and loss-of-function experiments revealed that miR-506 suppresses cell proliferation, invasion and adhesion of breast cancer cells. These findings are consistent with the reports by Arora et al (8).

To date, there has been no report on the molecular mechanisms underlying the role of miR-506 in breast cancer. In this study, we first identified IQGAP1 as a direct target of miR-506. IQGAP1 is a scaffold protein which has ubiquitous

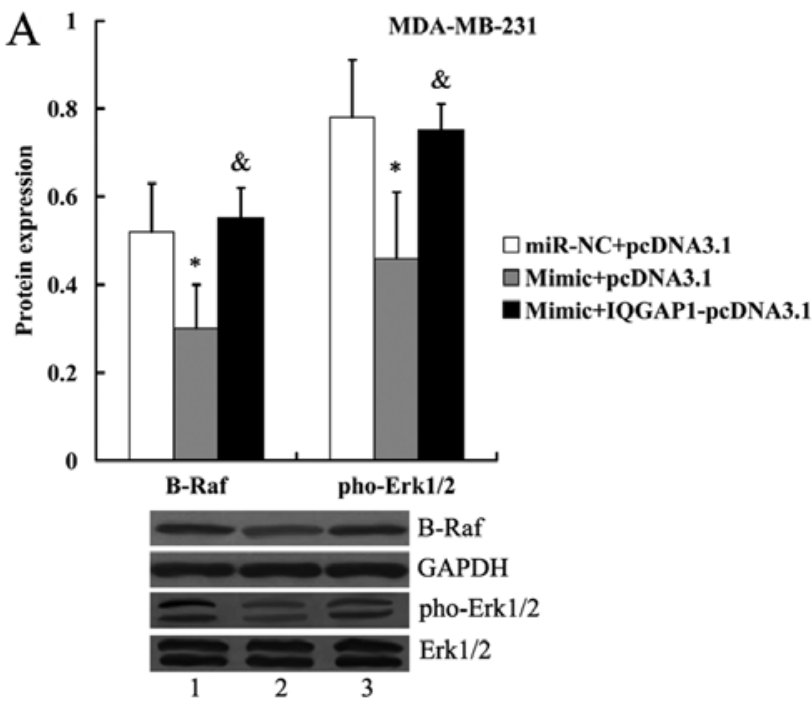

B

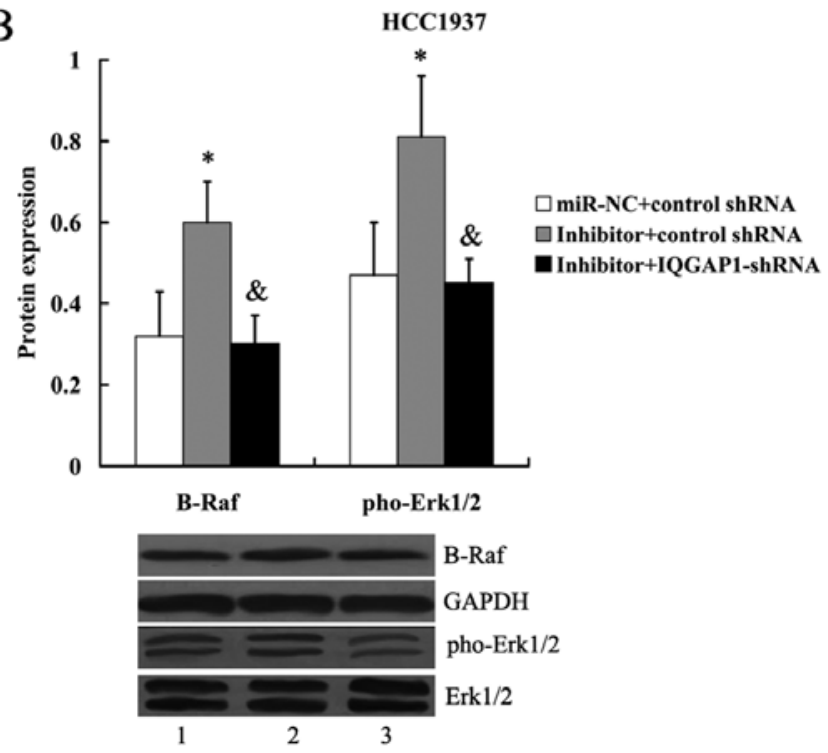

Figure 12. IQGAP1 attenuates the effect of miR-506 on B-Raf and pho-Erk1/2 expression. (A) Expression of B-Raf and pho-Erk1/2 protein in MDA-MB-231 cells following transfection with the miR-506 mimic and IQGAP1-pcDNA3.1. Lane 1, miR-NC+pcDNA3.1; lane 2, mimic+pcDNA3.1; lane 3, mimic+IQGAP1-pcDNA3.1. " $\mathrm{P}<0.05$ compared with miR-NC+pcDNA3.1; ${ }^{\&} \mathrm{P}<0.05$ compared with mimic+pcDNA3.1. (B) Expression of B-Raf and pho-Erk1/2 protein in HCC1937 cells following transfection with the miR-506 inhibitor and IQGAP1 shRNA. Lane 1, miR-NC+pcDNA3.1; lane 2, inhibitor+control shRNA; lane 3, inhibitor+ IQGAP1-shRNA. "P $<0.05$ compared with miR-NC+control shRNA; ${ }^{\&} \mathrm{P}<0.05$ compared with inhibitor+control shRNA. The expression of B-Raf was normalized to GAPDH, while the expression of pho-Erk1/2 was normalized to total Erk1/2. NC, negative control; IQGAP1, IQ motif containing GTPase activating protein 1; Erk, extracellular signal regulated kinase; pho, phosphorylated.

expression $(14,15)$. It contains multiple protein-interacting domains, including one calponin homology domain, one Ras-GAP-related domain, a polyproline binding domain and four calmodulin-binding motifs. IQGAP1 interacts with components of the cytoskeleton, the intercellular adhesion complex, and several signaling molecules, thus, having roles in many different aspects of cell physiology (16). 
IQGAP1 has attracted attention because IQGAP1 plays important roles in the control of cell adhesion, polarization and migration (17). Overexpression of IQGAP1 has been shown in glioblastoma, hepatocellular carcinoma, lung and pancreatic cancer (18-22). IQGAP1 expression is also associated with the development of breast cancer $(23,24)$. In the present study, we demonstrated that IQGAP1 expression is regulated by miR-506. Furthermore, IQGAP1 could attenuate the effect of miR-506 on breast cancer cell proliferation, invasion and adhesion.

The ERK mitogen-activated protein kinase (MAPK) pathway signaling is downstream of IQGAP1 (25), and this pathway was found to be repressed by miR-506 in the present study. There is a direct interaction between IQGAP1 and B-Raf, which modulates the activation of B-Raf (26). Raf was able to phosphorylate and activate the dual specificity protein kinases MEK1 and MEK2, thus leading to the phosphorylation of Erk1 and Erk2 (27). In addition, it has been demonstrated that IQGAP1 contributes to the regulation of epidermal growth factor (EGF)-stimulated ERK activity $(28,29)$. The $\mathrm{Ras} / \mathrm{Raf} / \mathrm{MEK} / \mathrm{ERK}$ cascade is suggested to play vital roles in several fundamental cellular activities (30). In the present study, we first proved that miR-506 represses the activation of B-Raf and Erk1/2, at least partially, by downregulation of IQGAP1.

Collectively, these findings revealed that miR-506 expression is downregulated in breast cancer and associated with the tumor stage. miR-506 inhibits breast cancer cell proliferation, invasion and adhesion, as well as the ERK MAPK pathway, at least partially, by directly downregulating IQGAP1. The miR-506/IQGAP1/ERK pathway may be a novel therapeutic target in breast cancer.

\section{Acknowledgements}

The present study is supported by the Jilin Province Key Scientific and Technical Project (no. 20150204081SF), China.

\section{References}

1. Badar F, Faruqui ZS, Ashraf A and Uddin N: Third world issues in breast cancer detection. J Pak Med Assoc 57: 137-140, 2007.

2. Jamal S, Mamoon N, Moghal S, Mushtaq S and Luqman M: Carcinoma breast: A histopathological audit. J Coll Physicians Surg Pak 16: 117-119, 2006.

3. Jemal A, Bray F, Center MM, Ferlay J, Ward E and Forman D: Global cancer statistics. CA Cancer J Clin 61: 69-90, 2011.

4. Chaffer CL and Weinberg RA: A perspective on cancer cell metastasis. Science 331: 1559-1564, 2011.

5. Ambros V: The functions of animal microRNAs. Nature 431: 350-355, 2004.

6. Nelson KM and Weiss GJ: MicroRNAs and cancer: Past, present, and potential future. Mol Cancer Ther 7: 3655-3660, 2008.

7. Liu G, Sun Y, Ji P, Li X, Cogdell D, Yang D, Parker Kerrigan BC, Shmulevich I, Chen K, Sood AK, et al: MiR-506 suppresses proliferation and induces senescence by directly targeting the CDK4/6-FOXM1 axis in ovarian cancer. J Pathol 233: 308-318, 2014.

8. Arora H, Qureshi R and Park WY: miR-506 regulates epithelial mesenchymal transition in breast cancer cell lines. PLoS One 8: e64273, 2013.
9. Wen SY, Lin Y, Yu YQ, Cao SJ, Zhang R, Yang XM, Li J, Zhang YL, Wang YH, Ma MZ, et al: miR-506 acts as a tumor suppressor by directly targeting the hedgehog pathway transcription factor Gli3 in human cervical cancer. Oncogene 34: 717-725, 2015.

10. Streicher KL, Zhu W, Lehmann KP, Georgantas RW, Morehouse CA, Brohawn P, Carrasco RA, Xiao Z, Tice DA, Higgs BW, et al: A novel oncogenic role for the miRNA-506-514 cluster in initiating melanocyte transformation and promoting melanoma growth. Oncogene 31: 1558-1570, 2012.

11. Buffa FM, Camps C, Winchester L, Snell CE, Gee HE, Sheldon H, Taylor M, Harris AL and Ragoussis J: microRNA-associated progression pathways and potential therapeutic targets identified by integrated mRNA and microRNA expression profiling in breast cancer. Cancer Res 71: 5635-5645, 2011

12. Zhao Y, Liu H, Li Y, Wu J, Greenlee AR, Yang C and Jiang Y: The role of miR-506 in transformed 16HBE cells induced by anti-benzo[a]pyrene-trans-7,8-dihydrodiol-9,10-epoxide. Toxicol Lett 205: 320-326, 2011.

13. Wang Y, Cui M, Sun BD, Liu FB, Zhang XD and Ye LH: MiR-506 suppresses proliferation of hepatoma cells through targeting YAP mRNA 3'UTR. Acta Pharmacol Sin 35: 1207-1214, 2014.

14. Weissbach L, Settleman J, Kalady MF, Snijders AJ, Murthy AE, Yan YX and Bernards A: Identification of a human rasGAPrelated protein containing calmodulin-binding motifs. J Biol Chem 269: 20517-20521, 1994.

15. Hart MJ, Callow MG, Souza B and Polakis P: IQGAP1, a calmodulin-binding protein with a rasGAP-related domain, is a potential effector for cdc42Hs. EMBO J 15: 2997-3005, 1996

16. Noritake J, Watanabe T, Sato K, Wang S and Kaibuchi K: IQGAP1: A key regulator of adhesion and migration. J Cell Sci 118: 2085-2092, 2005.

17. White CD, Brown MD and Sacks DB: IQGAPs in cancer: A family of scaffold proteins underlying tumorigenesis. FEBS Lett 583: 1817-1824, 2009.

18. Johnson M, Sharma M and Henderson BR: IQGAP1 regulation and roles in cancer. Cell Signal 21: 1471-1478, 2009.

19. Lu SH, Jiang XJ, Xiao GL, Liu DY and Yuan XR: miR-124a restoration inhibits glioma cell proliferation and invasion by suppressing IQGAP1 and $\beta$-catenin. Oncol Rep 32: 2104-2110, 2014.

20. Chen F, Zhu HH, Zhou LF, Wu SS, Wang J and Chen Z: IQGAP1 is overexpressed in hepatocellular carcinoma and promotes cell proliferation by Akt activation. Exp Mol Med 42: 477-483, 2010.

21. Nakamura H, Fujita K, Nakagawa H, Kishi F, Takeuchi A, Aute I and Kato H: Expression pattern of the scaffold protein IQGAP1 in lung cancer. Oncol Rep 13: 427-431, 2005.

22. Wang XX, Li XZ, Zhai LQ, Liu ZR, Chen XJ and Pei Y: Overexpression of IQGAP1 in human pancreatic cancer. Hepatobiliary Pancreat Dis Int 12: 540-545, 2013.

23. Erdemir HH, Li Z and Sacks DB: IQGAP1 binds to estrogen receptor- $\alpha$ and modulates its function. J Biol Chem 289: 9100-9112, 2014.

24. Jadeski L, Mataraza JM, Jeong HW, Li Z and Sacks DB: IQGAP1 stimulates proliferation and enhances tumorigenesis of human breast epithelial cells. J Biol Chem 283: 1008-1017, 2008.

25. Brown MD and Sacks DB: IQGAP1 in cellular signaling: Bridging the GAP. Trends Cell Biol 16: 242-249, 2006.

26. Ren JG, Li Z and Sacks DB: IQGAP1 modulates activation of B-Raf. Proc Natl Acad Sci USA 104: 10465-10469, 2007.

27. Schaeffer HJ and Weber MJ: Mitogen-activated protein kinases: Specific messages from ubiquitous messengers. Mol Cell Biol 19: 2435-2444, 1999.

28. Roy M, Li Z and Sacks DB: IQGAP1 is a scaffold for mitogenactivated protein kinase signaling. Mol Cell Biol 25: 7940-7952, 2005.

29. Roy M, Li Z and Sacks DB: IQGAP1 binds ERK2 and modulates its activity. J Biol Chem 279: 17329-17337, 2004.

30. McCubrey JA, Steelman LS, Chappell WH, Abrams SL, Wong EW, Chang F, Lehmann B, Terrian DM, Milella M, Tafuri A, et al: Roles of the Raf/MEK/ERK pathway in cell growth, malignant transformation and drug resistance. Biochim Biophys Acta 1773: 1263-1284, 2007. 